\title{
ATLAS CHRONICLE: DEVELOPMENT AND VERIFICATION OF A SYSTEM FOR PROCEDURAL GENERATION OF STORY-DRIVEN GAMES
}

\author{
Elizabeth A. Matthews ${ }^{1}$ and Juan E. Gilbert ${ }^{2}$ \\ ${ }^{I}$ Washington and Lee University, USA \\ ${ }^{2}$ University of Florida, USA
}

\begin{abstract}
Procedural Content Generation for Games is the application of computers to generate game content such as landscapes, vegetation, or maps. This paper proposes a system (Atlas Chronicle) for the generation of world maps for games that feature story-driven locations. The system uses a combination of story abstraction, graphs, physics simulation, Perlin noise, interpolation, and climate mapping to generate 2D tile-based maps given a basic story structure. To measure the fitness of the levels generated, a user study was performed using established game enjoyment metrics. The study utilized three different content generation approaches for comparison. Eighteen participants (11 Male, 7 Female), aged between 18 and 24 years old were screened based on their prior gaming experience. Results show that levels generated by Atlas Chronicle resulted in statistically similar responses as for the manually designed and static levels.
\end{abstract}

\section{KEYWORDS}

Procedural Generation, Video Games, Video Game Enjoyment

\section{INTRODUCTION}

\subsection{Motivation}

One of the efforts towards creating more enjoyable games involves the generation of content for said games. Traditionally, this content has been generated manually by human game designers and level designers. However, the two major problems with manual content generation for games are the expense (Takatsuki, 2007) and the fact that it does not scale (Iosup, 2011). Procedural Content Generation for Games (PCG-G) is the application of computers to generate game content and select the enjoyable items for use in games (Hendrikx et al., 2013).

Procedural Content Generation (PCG), or Procedural Generation (PG) for short, is the generation of virtual content by computers, typically through a human-defined procedure. As games get larger and more expansive, a cheaper and faster approach to generating the game content is highly desirable. PG has been utilized in games for a long time, starting with one of the earliest games, Rogue (Epyx, 1980).

Games with PG can have a minimal amount of generated content, but the most prevalent and noticeable forms are repeated-play variations. Games like Diablo (Blizzard North, 1996) have the same storyline each time the game is started by a gamer, but the dungeons in which the player progresses through the story are procedurally generated. As a result, each time the game is played, the exploration is different.

Traditionally, story-driven games have had minimal or compartmentalized procedural generation. Due to the innate relationship between the world map layout of locations of interest and the story to be told, the map is difficult to change without taking these story restrictions into account. The system described in this paper proposes a method that keeps the story structure intact but randomizes the directions one must travel to progress in the story. An abstracted story graph is used to implicitly build a map around the story locations. 


\subsection{Related Work}

Procedural Generation has been utilized to create enjoyable games since the early 1980s (Epyx, 1980). The more traditional approach has been to generate a multitude of options independently of the user, while a more recent approach has been to dynamically generate the content based on the user's gameplay.

The procedurally generated content ranges in size and scope in the final game. Most commonly created are "game bits," which are the basic units that compose a game (e.g. textures or sounds), or "game spaces," which are the navigational world/space in which the player exists (Hendrikx et al., 2013). The systems which create the procedural content range from tools and materials for game designers to utilize in their normal workflow to independent designers and domain experts which fully design and evaluate the game (Khaled et al., 2013). The Infinite Mario Competition is frequently cited by some of the PG researchers discussed in this section and has been one of the main motivators for discovering new PG techniques (Shaker et al., 2011).

There are two important approaches in PG techniques: Independent Procedural Content Generation (IPCG) generates content without context from the user, and Experience Driven Procedural Content Generation (EDPCG) generates content based on a user's performance and play style in the game. Independent Procedural Content Generation (IPCG) is the most popular version of PG, despite a recent rise in interest in research in EDPCG. IPCG generates many content variations, given a certain structure, then selects a variation for each play-through. The generation does not take input from the user. IPCG can be used to generate content from quests or puzzles (Pereira et al., 2016; Barros et al., 2016), platformer levels as in Spelunky or Mario (Baghdadi et al., 2015; Shaker et al., 2012; Yu and Hull, 2009), 3D terrain (Beckham and Pal, 2017; Frade et al., 2012), maps or mazes (Moore Jr, 2015; Togelius et al., 2013), creative sandbox games like Minecraft (Patrascu and Risi, 2016; Mojang, 2011), or infinite style games (Saltsman, 2009; Henschke et al., 2012).

\section{METHODOLOGY}

Story-driven games, often in the category of Role-Playing Games (RPGs), are one of the game categories that PG has barely touched due to the intertwined nature of the game world's map and the game story. We developed a system, Atlas Chronicle, that relies on the nature of RPG stories in which there is a partial order to the locations to visit. Further, the relative distances between story-based locations of interest remain within story-defined constraints of minimum and maximum distance and connectivity.

If a system can calculate a controlled randomization of locations for each location visited in the game that satisfies the minimum and maximum distance between the locations as defined by the story, one can infer the world around these locations to build a full map. The development of Atlas Chronicle finds this set of locations using a physical model of the game graph. Atlas Chronicle was developed in Python (2020) with the use of the PyGame module (PyGame, 2020) for visual and interactive aspects.

\subsection{Story Abstraction}

In a story-driven RPG there are locations of interest (LOI). These LOI can be a town, cave, castle, or even a non-interactive terrain type, so the journey the player experiences travels through different terrains. An RPG story can be abstracted into LOI and distance restrictions between each LOI. These restrictions are a story-experience restriction of distance: Town A and Cave B must be between 10 and 15 kilometers of each other. Restrictions represent a minimum and maximum value connecting two LOI.

These restrictions can be of two types: traversable or non-traversable. Traversable means that the restriction between LOIs A and B doubles as a rule that A must directly connect to B via traversable terrain. In our example, Town A and Cave B connected by a traversable restriction means that the direct path between A and B must be connected by walk-able terrain. The "traversable" definition for terrain can change (see Section 2.4 for details). Sometimes LOI have restrictions between them, but not a direct traversable path (e.g., the final location must be at least 50 units away from the starting location). These non-traversable 6 restrictions do not need to block the path between LOI but prevent LOI from being too far or too close to each other. Therefore, a non-traversable restriction does not need to be covered by non-traversable terrain; it simply does not require that the path be explicitly traversable terrain. This property is to allow restrictions to connect LOI to restrict their distances without affecting the connectivity of the terrain. Figure 1a shows a simple visualization of three LOI with three restrictions. 


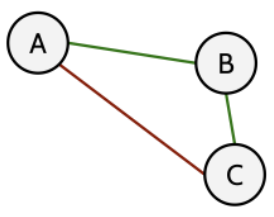

(a) Example of story abstraction with three LOI and three restrictions Green indicates traversable and red indicating not traversable

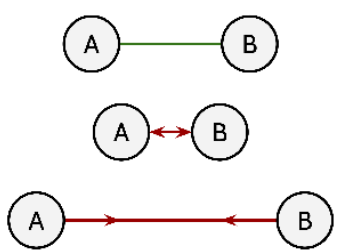

(b) Top to bottom slide spring states: no force applied, increase distance force applied, reduce distance force applied. Green indicates no forces applied and red indicating forces applied away (middle) and towards (bottom)

Figure 1. Story abstractions show LOI as circles and restrictions between LOI as lines

With abstraction, the world can be built around the story. The first step is to find some set of coordinates that satisfy all the restrictions in the connected graph of LOI. The settling of LOI in these satisfactory coordinates used a physics engine.

\subsection{Physics Engine}

A physics engine (Pymunk, 2019) applies forces on LOI as physical objects to push and pull the LOI into coordinates that satisfy the restrictions. Each LOI was represented as a physical object, and each restriction was made into a slide/spring combination. The connection functions as a free movement sliding joint when within valid distance measurements. If the connection distance/size is less than the minimum value the physical property is changed to a spring-style connection with a resting size of the minimum value. The forces applied will push the two connected LOI objects further away if the distance connecting them is too small. A similar physics interaction happens if the distance is larger than the maximum value with the force applied to bring the two LOI closer to each other. A visualization of this process is in Figure $1 b$.

With all LOI in the system and connected by slide-spring restrictions (weighted based on the story parameters), each LOI was given a random x,y coordinate and allowed to exist in a physical space. The system was allowed to simulate the forces until all the objects' velocity fell below a small threshold.

The use of a physics engine over other more deterministic algorithms was to account for human error in the story abstraction step. Future applications of the system will be available for game developers and a non-deterministic allowance will allow for imperfect systems to still create solutions.

\subsection{Terrain Generation}

Once each of the LOI has valid x,y coordinates that satisfy all restrictions to the best of the system's ability, the terrain around the LOI is generated. A randomized floodfill fills in terrain around the LOI. Each LOI was given a seed terrain, with each interact-able/town-based LOI creating four seeds around it of traversable terrain. The edges of the map were filled with void tiles, which are tiles that will be replaced in further iterations.

Figure 2 shows the process visually. Each of the seed tiles and edge void tiles were placed in a list, A, to be used in the floodfill sequence, Figure $2 \mathrm{a}$ and $2 \mathrm{~b}$. The floodfill algorithm, Figure 2c, was as follows:

1. Select a tile $t$ from the list of available tiles A

2. Populate list $\mathrm{T}$ of possible floodfill adjacent tiles from $\mathrm{t}$

a. Check all cardinal directions North, South, East, West of $t$

a. Add tile to $\mathrm{T}$ if it is unfilled and viable

3. IF no tiles are in $\mathrm{T}$, remove $\mathrm{t}$ from $\mathrm{A}$ and return to step 1

4. ELSE select one tile $t^{1}$ randomly from $T$

5. Fill $\mathrm{t}^{1}$ with the same tile type as $\mathrm{t}$ and add $\mathrm{t}^{1}$ to $\mathrm{A}$

6. Repeat steps until A is empty

Viable means that, in the case of void tiles, attempting to fill the tile cannot cross a boundary along the line of any traversable restriction. Any two LOI connected by traversable restrictions will have a map that connects them via traversable terrain. The final result was a map of traversable ("land"), not traversable ("mountain" or "water"), and void tiles, Figure $2 \mathrm{~d}$. 


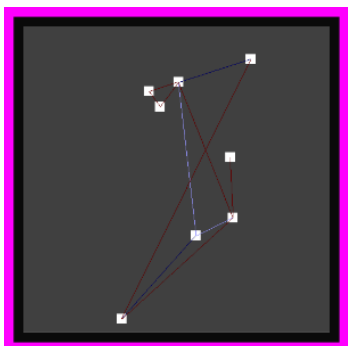

(a) Coordinates determined with physics engine

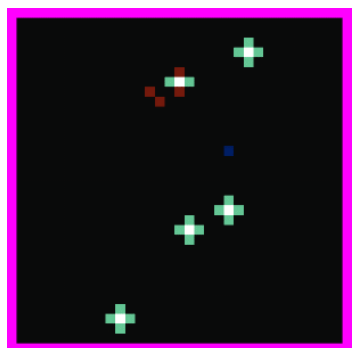

(b) Map with seed tiles based on LOI definitions

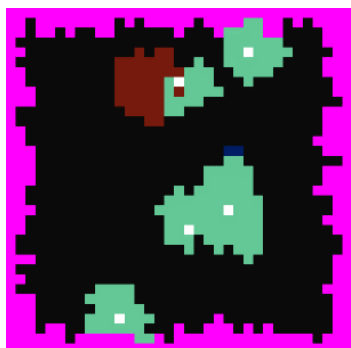

(c) Halfway through the flood-fill process

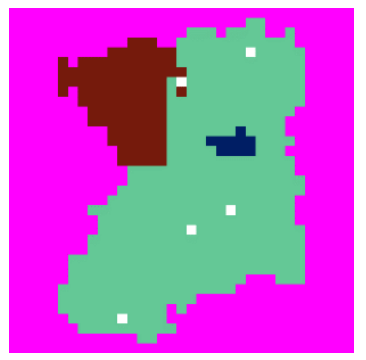

(d) A final possible map given LOI and restrictions

Figure 2. The process from the coordinates generated by the space manager to 2D map. Void tiles are pink, traversable terrain is green. Non-traversable appears in two formats: mountains (maroon) and water (blue)

\subsection{Recursive Process}

The next step repeated the process from Section 2.3 recursively. The traversable map generated, Figure $2 \mathrm{~d}$, is called a field. A field is a subset of what makes a continent, which is a subset of a world. A field is a set of LOI separated by traversable or not traversable terrain. A continent consists of fields separated by not traversable terrain. A world contains continents separated by water.

The next iteration for creating a continent repeated the process with fields in place of the LOI (Figure 3). The fields were connected by restrictions, placed in a physics engine randomly and allowed to settle (Figure 3a). The floodfill has a similar process with void tiles around the edge, but the floodfill seed tiles were mountains instead of landfill (Figure 3b), to ensure the two or more fields were connected by mountains, thus creating a single continent (Figure 3c and 3d). Figure 3e shows the climate to terrain mapping, covered later in this section.

The final recursive step repeated the process with continents instead of LOI (Figure 4a). Instead of floodfilling a third time, for this study's purposes the map is complete, and the unfilled areas were filled with ocean/water tiles (Figure 4b).

\subsection{Terrain Mapping}

After the continents were formed, the system mapped terrain types onto the landfill tiles (Matthews and Malloy, 2012). A climate was assigned to each LOI. Climates are made of a value between 0.0 and 1.0 for both temperature and humidity. A climate map was made by first creating seed values from each LOI and an average value was added to the edges (Figure 5a) to enable interpolation (SciPy, 2019) to the edges of the map. Then two interpolation maps were generated to interpolate the climate values in the unassigned tiles between LOI, one for each temperature and humidity, visualized as blue and red respectively in Figure 5b. Noise (noise 1.2.2, 2019) was added for a more natural shape of terrains in Figure $5 \mathrm{~d}$. Then the climate was mapped to a corresponding terrain type based on a look-up process called a Terrain Boundary Map (TBM) in Figure 6.

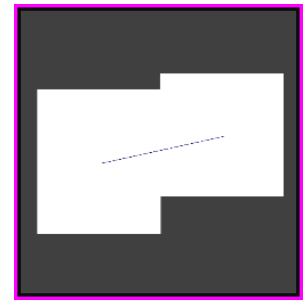

(a) Physics engine with two fields

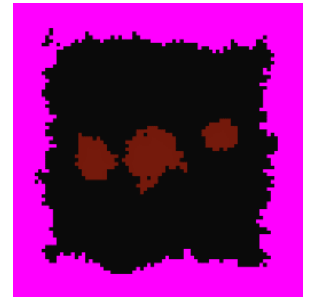

(b) Halfway through floodfill process

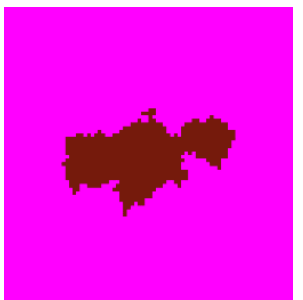

(c) Final mountain shape created

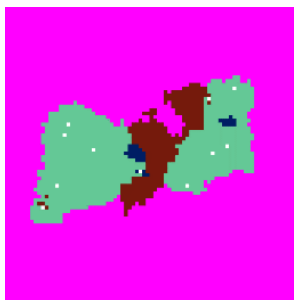

(d) Placement of fields using $3 \mathrm{a}$

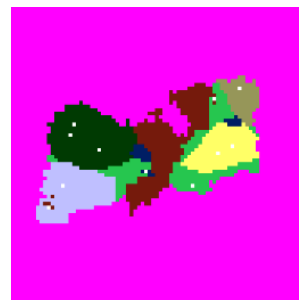

Climate mapping (Figure 5) applied

Figure 3. Recursive process for continent ending with one possible continent generated 


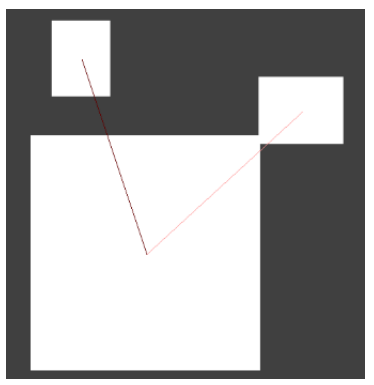

(a) Physics engine with three continents

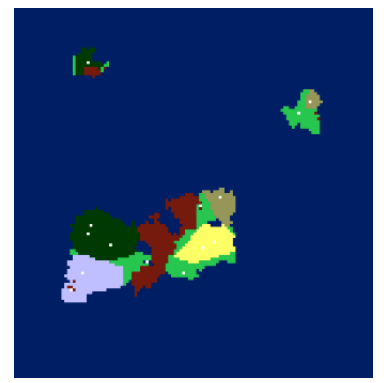

(b) Final possible world creation

Figure 4. Recursive process for world ending with one possible world generated

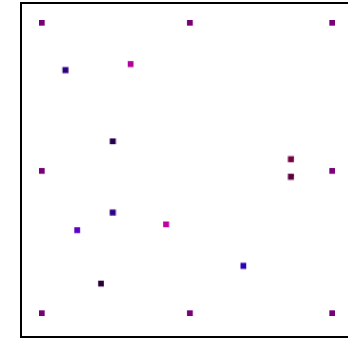

(a) Seed and edge values

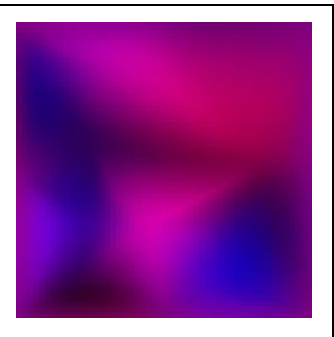

(b) Interpolation

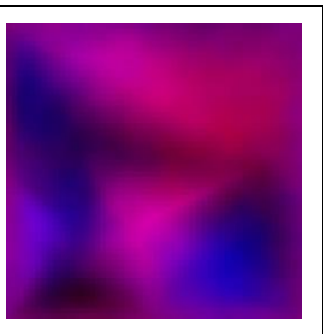

(c) Noise added

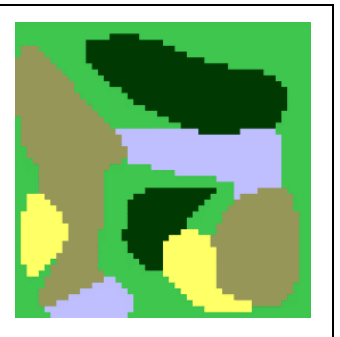

(d) Terrain mapping

Figure 5. An example of mapping seeded climate values to a full map via interpolation. The final steps translate the two-dimensional climate to a terrain via the Terrain Boundary Map (Figure 6)

The system achieved the climate to terrain mapping by creating a nearest neighbor map to the designer's specifications, the TBM (Matthews and Malloy, 2012). For this study the map seen in Figure 6 was used, but any amount of terrain values can be used. The values for temperature and humidity were used as a $\mathrm{x}, \mathrm{y}$ coordinate look-up in the nearest neighbor map, which converted the two values into a single terrain type. To create more organic looking separations between terrains from the interpolation step, a small amount of noise was added to the climate map before TBM look-up.

\subsection{Testing Game Engine}

The intended run-time of a normal RPG is 40+ hours, which was not feasible for a rapid, repeated test in a user study. Instead, for testing the procedural generation in RPGs used a minimal game engine and storyline created for the study. The engine was also programmed in Python/Pygame, seen in Figure 7. The game engine only contained the minimal elements to progress through the story: Overworld movement, locked terrain type travel, text boxes/interactable locations of interest, gating items obtained from locations of interest.

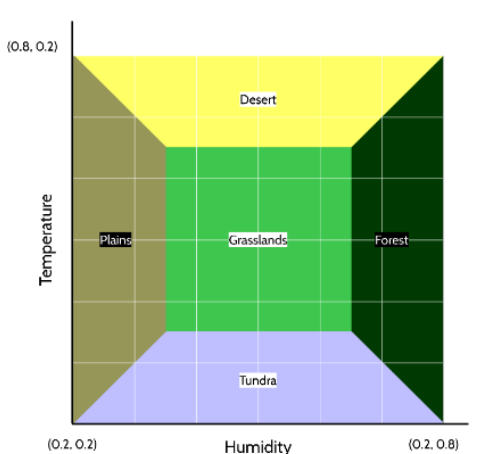

Figure 6. Terrain Boundary Map used in examples for testing game

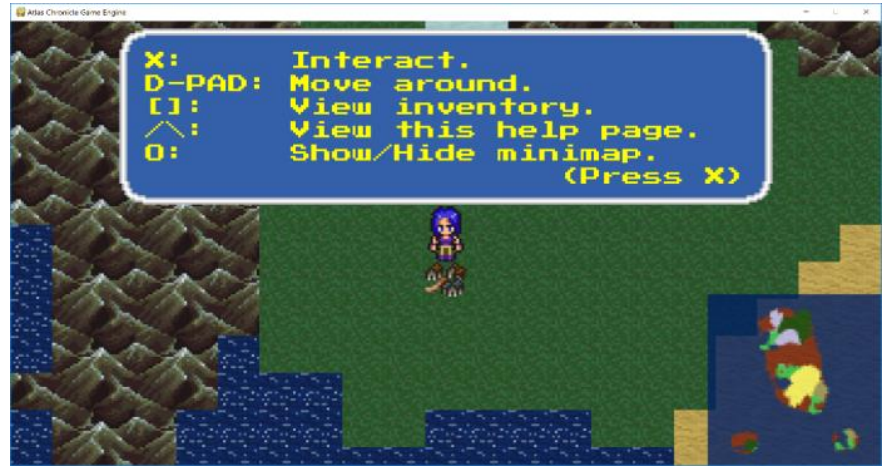

Figure 7. Example gameplay of the 2D RPG testing engine for Atlas Chronicle 
The gating items were given to the player once they interacted with a location containing the item (e.g. The Desert Palace gives the player the Crystal of Courage). Certain locations would not permit further progression until the correct gating item was obtained. These gating items were used to simulate the traditional gating behavior of RPGs, where some challenge is required to "open up" the next exploration area. The map generated by Atlas Chronicle was converted to a two-dimensional game via Python and PyGame. The game engine kept track of the time elapsed in-game and would pause the game automatically at timed intervals to collect the survey information. The game would only be allowed to continue after the participant completed the survey.

\subsection{User Study}

The terms "replay value" or "replayability" are often used when describing the benefit of procedural generation in games. We define the concept of replay value formally, with respect to the use of this term to describe video game enjoyment, as the rate of enjoyment decline over time spent playing the game. There are established methods for measuring enjoyment in video games using subjective style questionnaires. One frequently used questionnaire, the Game Experience Questionnaire (IJsselsteijn et al., 2008), has been thoroughly tested (Kätsyri et al., 2013; Mekler et al., 2014; Johnson et al., 2015) and is easily applied to measuring enjoyment in PG applications. The Fang et al. Questionnaire was also used because it is reliable way to measure Affect (Fang et al., 2010). Both the GEQ and Fang et al. Questionnaire were used for this study.Participants were selected from applicants with some expressed interest in playing video games and were screened using a brief screening form. The screening form asked for the applicant's self-reported gamer level and to select which types of games they regularly enjoy. Applicants who selected RPGs as a game type they regularly enjoyed were selected to participate. Also selected were applicants who labeled themselves as an expert or frequent gamer. A total of 18 participants (11 Male, 7 Female), aged between 18 and 24 years old (average age of 20), were selected for the study.

The user study included two phases: phase 1 evaluated an infinite runner type game that was generated by a separate procedural generation system (Matthews, 2019). Phase 2, described in this paper, measured enjoyment of static, manual, and PG (Atlas Chronicle) versions of the RPG. The same participants were used for both phases to allow a within-subjects comparison. Due to the length of time necessary for each phase, the two phases were accomplished on separate days, with some participants performing phase 2 before phase 1 .

Participants were assigned the three content generation formats in random order: static, manual, or procedural. For the procedural and manual formats, each successive game that was played was different, with the creation of the content created by the computer or human designers, respectively. The static format game was replayed using a single created level for all games played.

Participants completed successive play-throughs of the RPG for half an hour per generation type. Due to the rapid iterative process, the subjective questionnaire enjoyment measurements were recorded exactly five times instead of after each game iteration. The RPG would play for 6 minutes before displaying the questionnaire, repeated for a total of five times per generation type. The time was decided after a pilot test determined the average play time a single game of the simplified RPG would take. The data collection was contained within the computer rather than on paper or at a different digital location to minimize player removal from the game-play experience. After the fifth questionnaire was completed the participant was informed to come find the observer and take their stretch break (a brief walk and restroom access). After the break, the participant was switched to the next content generation type.

\subsection{Analysis}

The independent variables were the three level generation methods: static, manual, and procedural. The dependent variables were the subjective questionnaire values: The 14 questions from the In-Game GEQ Module and Affect as measured by the 5 questions in the Fang et al. Questionnaire. User responses were recorded periodically during each Game/Generation section. The responses were on a Likert scale from 0 to 4 . To detect patterns, a contingency table was built upon varying factors and then the results were tested for statistical significance. 


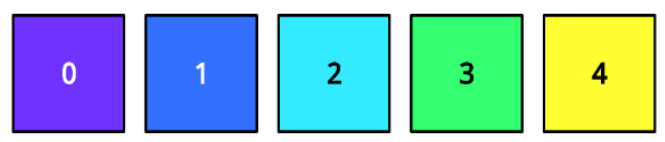

Figure 8. Color key used in the subjective contingency tables

The first test applied on a contingency table was the Chi-Squared test. GEQ03, "I felt bored," was examined by segment number. The p-value was less than 0.01 , therefore, the null hypothesis was rejected, and it was concluded that there was a relationship between segment number and response values for GEQ03. The questionnaire data are discrete rather than continuous; therefore, nonparametric tests were needed. Due to repeated significance testing, the chances of a Type I error ("false positive" or rejection of a true null hypothesis) are elevated, and hence significance values for each test must be adjusted to reflect the true probability under the null hypothesis. The Holm-Bonferroni p-value adjustment method was applied to counteract this effect.

For visualization, colors are used to distinguish the different response values, with the color key labeled in Figure 8. Seen in Figure 9a, the numerical totals are represented as a proportion of the total responses per segment. A larger segment indicates a larger percentage of the total. The upper x-axis is used to indicate the segment number and the lower $\mathrm{x}$-axis is used to indicate the pairwise results. The chi-squared p-value is in the upper right and the Wilcoxon results as labels are on the bottom axis.

The results from the chi-squared test and nonparametric pairwise tests indicated that that boredom, as measured by GEQ03, increased significantly from the first segment (Segment 1) to the last segment (Segment 5) during the test.

Given that there appeared to be a general increase in boredom over time, the analysis compared the early and late responses, focusing on the survey responses from segment 1, collected after the participants had played the game for the earlier designated amount of time, and segment 5, which were collected after playing the generation type for the full amount of time (Figure 9b). Generally, the pairwise analysis indicates that segment 1 for the manual designs was "better" than segment 5 of the static and procedural designs regarding negative affect scores. But all other categories were too similar to differentiate. However, one can conclude is that the segment 1 (start) values for all three generation types were similar, and all three segment 5 (end) values were similar. The conclusion is that procedural was no better and no worse than any other generation approach.

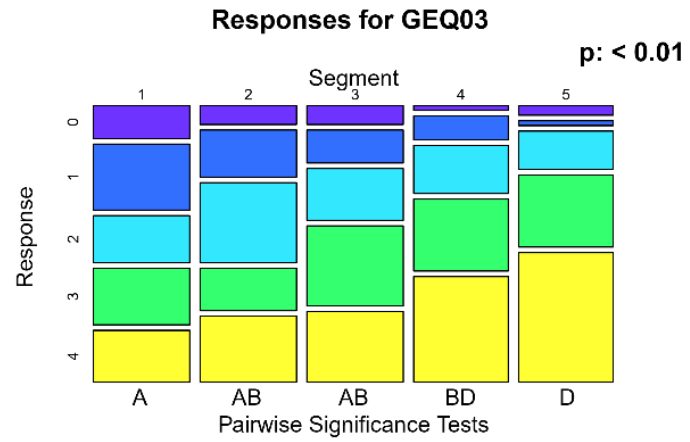

(a) Overall responses for boredom over all five segments

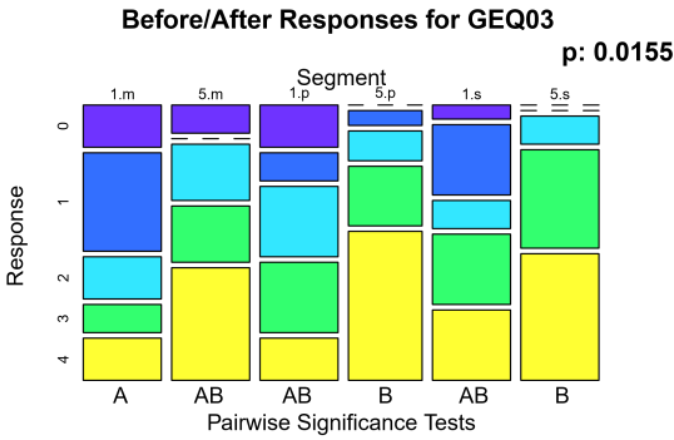

(b) Responses at the start and end of the test, pooled by generation type

Figure 9. Visual contingency tables using the color key from Figure 8 for the GEQ question "I felt bored." Y-axis is the response value from 0 (not at all) to 4 (extremely). $\mathrm{X}$-axis on the top labels the segment for which the response was recorded, with $\mathrm{m} / \mathrm{p} / \mathrm{s}$ for manual/procedural/static. Bottom X-axis labels the pairwise analysis: columns which do not share a letter are significantly different 


\section{CONCLUSION}

Based on the analysis, the boredom question from the GEQ measured a decline in enjoyment, over time. However, when comparing a before and after approach between the generation types, the procedurally generated test set performed the same as the manual and static design sets. This does call into question the claim of procedural generation increasing replay value, but for this research we can conclude that procedural generation is no better and no worse than static or manual generation approaches. According to our results, if a game designer wanted to have multiple, varied levels for their game, Atlas Chronicle would be a viable option because no enjoyment would be lost by using procedural generation, and procedural generation is faster and more cost-effective than manual procedures.

This study contributes to the analysis of procedural generation and enjoyment within video games. As such, the study was designed to minimize any external factors. The game engine utilized in this study was simplified to reduce confounding factors. A next step would perform the test on longer variations of a game utilizing the Atlas Chronicle map generation. Additionally, the story narrative used for testing Atlas Chronicle was simple due to the shorter variation of the game. Another avenue would be to examine the effects with a more compelling storyline. Participants were limited to college-aged individuals and the results may vary given different age ranges. The study measured a decline in enjoyment over time during a single-day session. Future studies should look into multiple-day studies, playing the game once per day. Future work could extend this research by adapting the definition to encompass the desire to replay a game as part of "replay value." Finally, the measurement tools utilized in this study were previously established enjoyment measurement tools, but not specifically tools to measure the type of enjoyment that procedural generation is said to bring to games. Future work must be done to examine how to measure "replay value" as affected by procedural generation in video games.

We revealed statistically relevant conclusions about the procedurally generated maps created by the Atlas Chronicle system. In summary, the maps generated were no different than human-designed maps in terms of retaining enjoyment over time. Participants experienced the same decline in enjoyment over time regardless of the content generation used.

\section{REFERENCES}

A. Iosup. Poggi: generating puzzle instances for online games on grid infrastructures. Concurrency and Computation: Practice and Experience, 23(2):158-171, 2011.

A. Saltsman. Canabalt (pc game). Adam Atomic, 2009.

ACM Transactions on Multimedia Computing, Communications, and Applications (TOMM), 9(1):1, 2013.

Blizzard North. Diablo (pc game), 1996.

C. Beckham and C. Pal. A step towards procedural terrain generation with gans. arXiv preprint arXiv:1707.03383, 2017.

C. Patrascu and S. Risi. Artefacts: Minecraft meets collaborative interactive evolution. In Computational Intelligence and Games (CIG), 2016 IEEE Conference on, pages 1-8. IEEE, 2016.

D. Johnson, P. Wyeth, M. Clark, and C. Watling. Cooperative game play with avatars and agents: Differences in brain activity and the experience of play. In Proceedings of the 33rd Annual ACM Conference on Human Factors in Computing Systems, pages 3721-3730. ACM, 2015.

D. Yu and A. Hull. Spelunky (pc game), 2009.

E. A. Matthews and B. A. Malloy. Incorporating coherent terrain types into story-driven procedural maps. In Meaningful Play, 2012: Designing and Studying Games that Matter, 2012.

E. A. Matthews. A Study of Techniques for Measuring Enjoyment in Video Games Containing Procedural Generation. PhD thesis, University of Florida, 72019.

E. D. Mekler, J. A. Bopp, A. N. Tuch, and K. Opwis. A systematic review of quantitative studies on the enjoyment of digital entertainment games. In Proceedings of the SIGCHI Conference on Human Factors in Computing Systems, pages 927-936. ACM, 2014.

Epyx. Rogue. Game [PC], 1980.

G. A. Barros, A. Liapis, and J. Togelius. Playing with data: Procedural generation of adventures from open data. In DiGRA/FDG, 2016.

J. Kätsyri, R. Hari, N. Ravaja, L. Nummenmaa, et al. Just watching the game ain’t enough: striatal fmri reward responses to successes and failures in a video game during active and vicarious playing, 2013. 
J. Togelius, M. Preuss, N. Beume, S. Wessing, J. Hagelbäck, G. N. Yannakakis, and C. Grappiolo. Controllable procedural map generation via multiobjective evolution. Genetic Programming and Evolvable Machines, 14 (2):245-277, 2013.

L. T. Pereira, C. Toledo, L. N. Ferreira, and L. H. Lelis. Learning to speed up evolutionary content generation in physics-based puzzle games. In Tools with Artificial Intelligence (ICTAI), 2016 IEEE 28th International Conference on, pages 901-907. IEEE, 2016.

M. Frade, F. F. de Vega, and C. Cotta. Automatic evolution of programs for procedural generation of terrains for video games. Soft Computing, 16(11):1893-1914, 2012.

M. Hendrikx, S. Meijer, J. Van Der Velden, and A. Iosup. Procedural content generation for games: A survey.

M. Henschke, D. Hobbs, and B. Wilkinson. Developing serious games for children with cerebral palsy: case study and pilot trial. In Proceedings of the 24th Australian Computer-Human Interaction Conference, pages 212-221. ACM, 2012.

Mojang. Minecraft (pc game), 2011.

N. Shaker, J. Togelius, G. N. Yannakakis, B. Weber, T. Shimizu, T. Hashiyama, N. Sorenson, P. Pasquier, P. Mawhorter, G. Takahashi, et al. The 2010 mario ai championship: Level generation track. IEEE Transactions on Computational Intelligence and AI in Games, 3(4):332-347, 2011.

N. Shaker, M. Nicolau, G. N. Yannakakis, J. Togelius, and M. O'neill. Evolving levels for super mario bros using grammatical evolution. In Computational Intelligence and Games (CIG), 2012 IEEE Conference on, pages 304-311. IEEE, 2012.

noise 1.2.2. noise 1.2.2. Website, 2019. URL https://pypi.org/project/noise/.

O. K. Moore Jr. Procedural content generation: Using ai to generate playable content. 2015.

PyGame. Pygame 1.9.6. Website, 2020. URL https://www.pygame.org/.

Pymunk. Pymunk 5.5.0. Website, 2019. URL http://www.pymunk.org/en/latest/.

Python. Python 3.7. Website, 2020. URL https://www.python.org/.

R. Khaled, M. J. Nelson, and P. Barr. Design metaphors for procedural content generation in games. In Proceedings of the SIGCHI Conference on Human Factors in Computing Systems, pages 1509-1518. ACM, 2013.

SciPy. Scipy 1.3.0. Website, 2019. URL https://www.scipy.org/.

W. Baghdadi, F. S. Eddin, R. Al-Omari, Z. Alhalawani, M. Shaker, and N. Shaker. A procedural method for automatic generation of spelunky levels. In European Conference on the Applications of Evolutionary Computation, pages 305-317. Springer, 2015.

W. IJsselsteijn, Y. de Kort, and K. Poels. The game experience questionnaire. Manuscript in preparation, 2008.

X. Fang, S. Chan, J. Brzezinski, and C. Nair. Development of an instrument to measure enjoyment of computer game play. Intl. Journal of Human-Computer Interaction, 26(9):868-886, 2010.

Y. Takatsuki. Cost headache for game developers. Website, 2007. URL news.bbc.co.uk/2/hi/business/ 7151961.stm. 